\title{
Learning Input Shaping Technique for Non-LTI Systems
}

\author{
Juyi Park and Pyung-Hun Chang \\ Department of Mechanical Engineering \\ Korea Advanced Institute of Science and Technology \\ 373-1, Kusung-dong, Taejon, Korea \\ phchang@mecha.kaist.ac.kr
}

\begin{abstract}
It is well known that a conventional Input Shaping Technique is not very effective in suppressing residual vibrations for non-LT'I systems, such as substantially nonlinear or timevarying systems. In an effort to increase the effectiveness for such systems, this paper presents a Learning Input Shaping Technique(LIST), which iteratively updates the parameters of the IST from the previous trials. Simulations are presented for 4 different cases: (1) when the natural frequency or damping of a system does not estimated well; (2) when a system has time varying vibration; (3) when a system has nonlinear flexibility; and (4) when a closed loop system includes the saturation limit in the loop. The experiments are made by using a six D.O.F industrial robot to evaluate the method. The results of both the simulations and experiments show that the residual vibrations become considerably smaller as iteration goes on, thereby demonstrating the effectiveness of the LIST.
\end{abstract}

\section{Introduction}

As is well known, the Input Shaping Technique (IST) does not suppress residual vibration of non-LTI - nonlinear or time varying - systems very well. The reason for this is that the principles of the IST are based on and suitable only for the Linear Time Invariant (LTI) system. Even a robust IST[1][2], which adopts an increased number of impulses at the cost of the increased time lag, is not so effective as expected for such systems.

In response to this difficulty, there have been many studies to improve the IST for non-LTI systems. Tzes and Yurkovich [3] proposed an on-line adaptive scheme which updated the IST parameters by using real time estimation in the frequency domain. By using this scheme, the residual vibration of pneumatic control system was suppressed. Bodson[5] proposed an adaptive scheme for tuning the parameters of the IST by using a stabilized recursive leastsquares algorithm. Rappole[6] used time varying IST parameters to suppress time varying vibration. Magee and Book[7][8] modified the IST to eliminate the first two modes of vibration in a large, flexible manipulator having position dependent parameters.

As another direction, the iterative learning schemes may well be considered for systems conducting repetitive tasks, which in fact take a substantial portion among the tasks that can be benefited with the IST. To our knowledge, there are few research taking this direction except an iterative learning scheme for the posicast technique developed by Noritsugu[4]. Being a posicast technique, however, it appears that this scheme is useful only for the step input.

In the context described thus far, this paper presents the iterative learning scheme based on the IST (LIST) as a remedy for eliminating residual vibrations of non-LTI systems conducting repetitive tasks. More specifically, the LIST iteratively updates the IST parameters: the magnitude and the time of each impulse input of impulse sequence. Hence, as the result of its nature, the LIST has some merits: First, since the learning process is performed on off-line base, the realtime computation is not required. Second, it does not require a dynamic model of the system. Third, the LIST can be used in conjunction with any type of input.

The paper is structured as follows: the following section describes the main idea and the algorithm of the LIST. In Section 3 the results of simulations are presented as examples. Section 4 presents the experimental results with a six D.O.F industrial robot. Finally in Section 5, conclusions are drawn.

\section{Iterative Learning Input Shaping Technique}

In 2.1, we will first represent the cause of the insufficient suppression of residual vibration, in terms of mismatches in the IST parameters; and then propose a quantitative measure to represent the amount of unsuppressed residual vibration. In 2.2 , follows our remedy, the LIST.

2.1. The response to the IST having improper parameters

Observing residual vibrations of various non-LTI systems with the IST being applied, one often finds the following trends: there exist a proper set of IST parameters for the effective suppression of residual vibrations; and the magnitude of the vibrations increase in proportion with the deviation of the IST parameters from their proper values ${ }^{1}$. These trends, already familar in LTI systems, enable us to represent the cause of the insufficient suppression of residual

${ }^{1}$ The validity of these trends is open to question for general non-LTI systems, and to prove it requires a rigorous analysis. 
vibration, in terms of mismatches in the IST parameters, as follows.

To this end, let us first examine how an LTI system is affected by the mismatches in IST parameters, by investigating the response of the following second-order system to the 2-impulse sequence.

$$
\frac{y(s)}{u(s)}=\frac{\omega_{n}^{2}}{s^{2}+2 \zeta \omega_{n} s+\omega_{n}^{2}}
$$

The 2-impulse sequence has four parameters: the magnitudes of impulses $A_{1}$ and $A_{2}$, and the appling times $T_{1}$ and $T_{2}$. In general, $T_{1}$ is fixed to 0 for the fastest response, and the $A_{2}$ is also fixed to $\left(1-A_{1}\right)$ to keep unit gain. Therefore there are only 2 independent parameters left: $A_{1}$ and $T_{2}$.

According to the study by Singer[1][2], the residual vibration due to the 2-impulse sequence vanishes if $A_{1}$ and $T_{2}$ are properly selectecl by the following equation.

$$
\begin{aligned}
A_{1 n} & =\frac{1}{1+e^{-\zeta \pi / \sqrt{1-\zeta^{2}}}} \\
T_{2 n} & =\frac{\pi}{\omega_{n} \sqrt{1-\zeta^{2}}}
\end{aligned}
$$

When $A_{1} \neq A_{1 n}$ or $T_{2} \neq T_{2 n}$, vibration does not vanish after $T_{2}$. There are nine possible cases depending on the combinations of the following conditions.

Condition $1 A_{1}>A_{1 n}$ or $A_{1}=A_{1 n}$ or $A_{1}<A_{1 n}$

Condition $2 T_{2}>T_{2 n}$ or $T_{2}=T_{2 n}$ or $T_{2}<T_{2 n}$

Among the nine cases, two examples are selected to examine the responses: $A_{1}=A_{1 n}$, and $T_{2}>T_{2 n}$ or $T_{2}<T_{2 n}$. Fig. 1 shows the responses of two cases. In the figure, the solid line represents the original vibration - the unit impulse response without the IST. The dotted lines are the resultant responses to the 2-impulse sequence. The upper plot shows the response for the case $T_{2}>T_{2 n}$, and the lower plot shows for the case $T_{2}<T_{2 n}$. It shows that there exist residual vibrations and phase differences compared to the original vibration in both cases.

As a measure representing the magnitude of the residual vibration, $M$ is defined as follows:

$$
M \equiv \int_{t_{i}}^{t_{f}}|y(t)| d t
$$

where $y(t)$ is any signal representing residual vibration such as position, velocity or acceleration. The range $t_{i} \leq t \leq t_{f}$ is the range in which the residual vibration is significant. The upper plot in Fig. 2 shows the variation of $M$ for the system (1) for $T_{2}$ varying with $A_{1}$ being fixed. The lower plot in Fig. 2 shows the phase difference, $\phi$, between the residual vibration and the original unit impulse response. In the Fig. 1, it is shown that the $\phi$ is different in the sign.
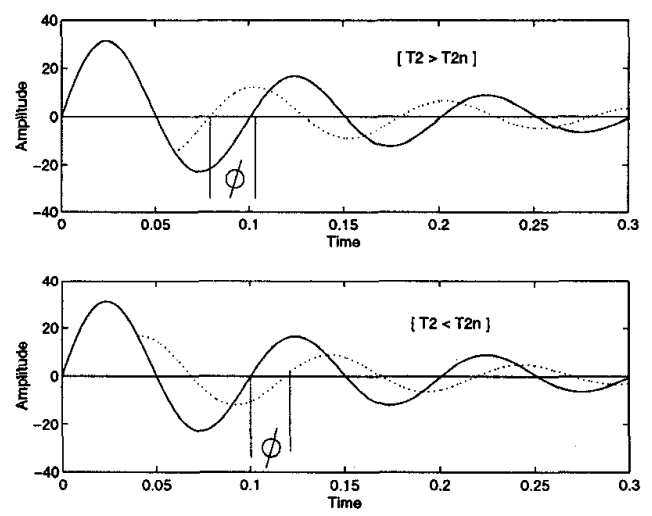

Figure 1: The responses to the 2-impulse sequence with inaccurate parameters; Solid lines: unit impulse responses without IST; Dotted lines: the resultant responses to the 2-impulse sequence with a large (upper plot) or a small (lower plot) $T_{2}$.
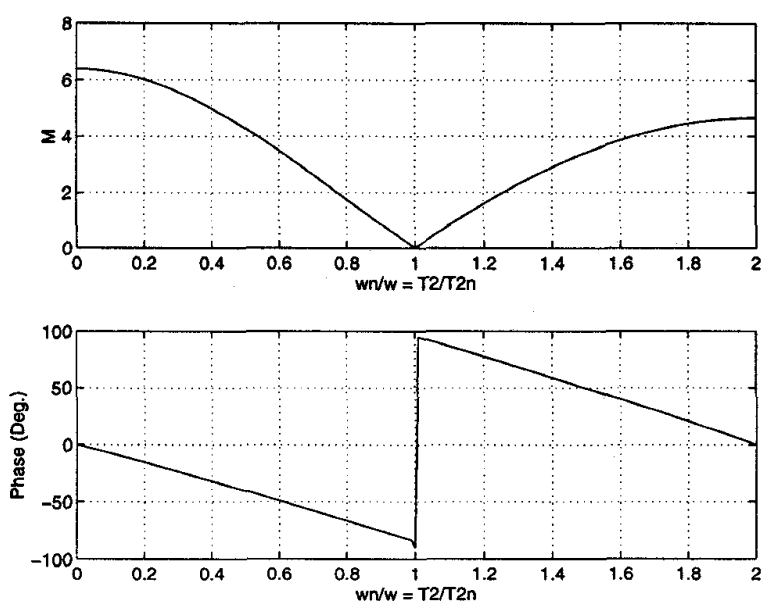

Figure 2: $M$ and $\phi$ for $T_{2}$ varying with $A_{1}$ being fixed.

The variation of $\phi$ for the variation of $T_{2}$ was plotted in Fig. 2.

In other cases, the shape of variation of the $M$ and $\phi$ for the variation of $T_{2}$ is not so different from those of above cases. Therefore the plots for other cases were omitted. The range of the $\phi$ is summarized in Table 1 for each case.

Thus far, we have examined how an LTI system is affected by the mismatches in IST parameters. More specifically, how $M$ and $\phi$ are related to mismatches in $A_{1}$ and $T_{2}$. For the non-LTI systems sharing these trends with LTI systems, the analysis above can be used to derive a learning scheme in 2.2 .

2.2. Learning Input Shaping Technique The iterative learning scheme updates the IST parameters by using the results of previous trials. $M$ and $\phi$ are obtained from the previous results, and then IST parameters are updated to minimize $M$. The algorithm is summarized 
Table 1: The ranges of $\phi$ for nine possible cases.

\begin{tabular}{|c|c|c|c|}
\hline & $A_{1}>A_{1 n}$ & $A_{1}=A_{1 n}$ & $A_{1}<A_{1 n}$ \\
\hline$T_{2}>T_{2 n}$ & $0<\phi<\frac{\pi}{2}$ & $0<\phi<\frac{\pi}{2}$ & $0<\phi<\pi$ \\
\hline$T_{2}=T_{2 n}$ & $\phi=0$ & $n o v i b$. & $\phi=\pi$ \\
\hline$T_{2}<T_{2 n}$ & $-\frac{\pi}{2}<\phi<0$ & $-\frac{\pi}{2}<\phi<0$ & $-\pi<\phi<0$ \\
\hline
\end{tabular}

as follows.

Step 1 Select an initial guess of $A_{k}$ and $T_{k}$, and obtain residual vibration, either by simulation or experiment.

Step 2 Fix $A_{k}$. Continue to simulate (or experiment), while varying $T_{k}$ until the $M$ has a minimum value.

Step 3 Set the best $T_{k}$ obtained at Step 2 fixed, and repeat the same procedure while varying $A_{k}$.

Step 4 Repeat step 2 and 3 until the $M$ has a minimum value.

The rule for updating parameters is determined by the relations of the values of parameters used in previous two trials. Fig. 3 illustrates the relation of two parameters. If both values are larger or smaller than the exact value, the secant method, a root finding method, is used. When one of the values is larger than the exact value, and the other is smaller, the weighted average value of the two parameters is used for the next trial.

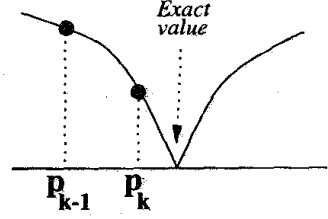

(a)

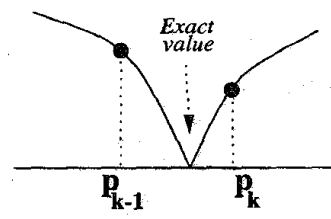

(b)
Figure 3: The relation of two parameters that were used in previous trials.

The following is the updating rule for the case of Fig. 3(a).

$$
p_{k+1}=\frac{M_{k-1}}{M_{k-1}-M_{k}}\left(p_{k}-p_{k-1}\right)+p_{k-1}
$$

Where $M_{j}$ means the $M$ of $j^{\prime}$ th trial, and $p_{j}$ means the parameter $A_{1}$ or $T_{2}$ of $j$ 'th trial. The following is the updating rule for the case of Fig. 3(b).

$$
p_{k+1}=\frac{M_{k-1} p_{k}+M_{k} p_{k-1}}{M_{k-1}+M_{k}}
$$

The initial guessing values that are needed to adopt the LIST initially can be obtained from the residual vibration without IST.

\section{Simulations}

Simulations are performed for the four examples. The followings are the systems and the methods for the simulations.

Case 1. When the natural frequency of a linear system is inaccurately estimated : for a 2 nd order LTI system, $\frac{y(s)}{u(s)}=\frac{k}{m s^{2}+b s+k}$, its simulation model is decided to

$$
m \ddot{x}+b \dot{x}+k(x-u)=0
$$

where $m=1, b=1, k=100$ and $u$ is the command input. The simulation starts when $\omega_{n}$ and $\zeta$ are estimated to be $20 \%$ larger than the exact value.

Case 2. When a linear system has time varying vibrations : the plant is the same as Eq. (7) except that a function of $t$ is substituted for the constant $m$.

$$
m(t) \ddot{x}+b \dot{x}+k(x-u)=0
$$

where $m(t)=1+t$ for $t<1$ and $m(t)=2$ for $t \geq 1$.

Case 3. When there exists a nonlinear spring : the plant is also the same as Eq. (7) except that a nonlinear spring is substituted for the constant $k x$.

$$
m \ddot{x}+b \dot{x}+k(x-u)^{3}=0
$$

Case 4. When there exists a saturation element : This system is illustrated in Fig. 4. The plant is the simple second-order system and the $\mathrm{P}$ controller is used as the closed loop controller. The saturation element exists in the block next to the $\mathrm{P}$ controller. In the real plant, it corresponds to the actuator limit.

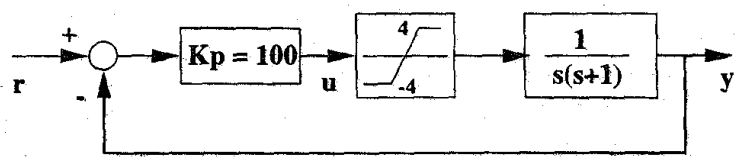

Figure 4: The shematic of a P-control system in which the saturation limit is included.

Fig. 5 and 6 show the results of all simulations using the LIST. This results are for the reference trajectories which have trapezoidal velocity profiles. The responses are shown in Fig. 5, and the change of the measure $M$ is presented in Fig. 6. It shows that the residual vibrations become smaller and smaller as the number of iterations increases. However, In the case 4 , the $M$ increased at 7 th iteration. It seems that the system has different characteristics from the relation shown in Fig. 2.

\section{Experiment}

The plant used in the experiment is a six D.O.F industrial robot shown in the schematic diagram of which is Fig. 7. 

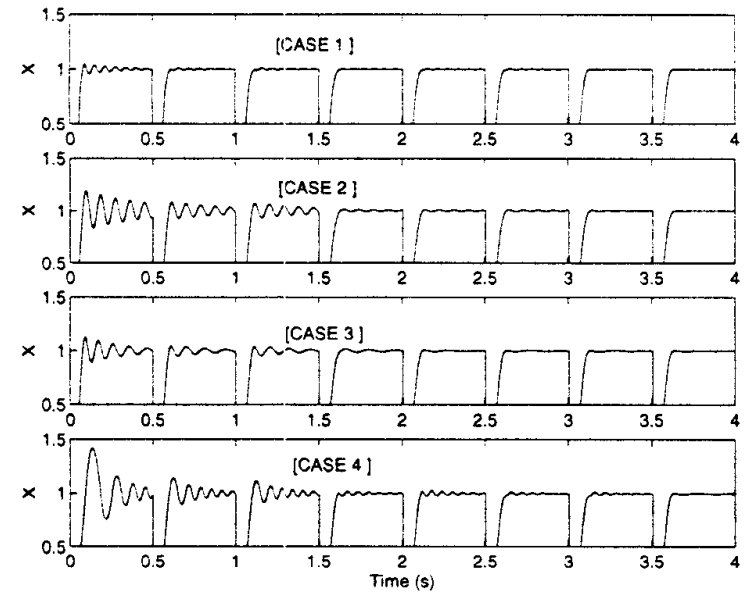

Figure 5: Simulation results: the change of residual vibrations. Residual vibrations become smaller and smaller as the number of iterations increases.
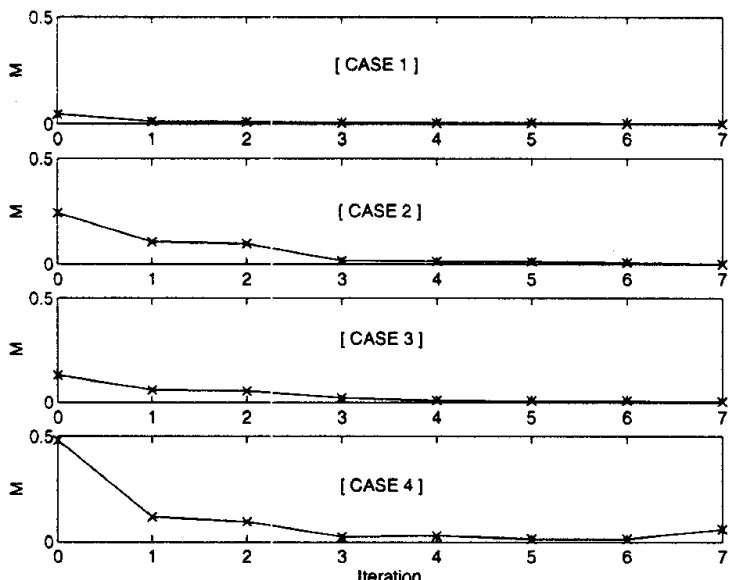

Figure 6: Simulation results: the $M$ decreses as the number of iterations increases.

The robot was designed to handle the spot welding gun in the production line of automobiles and to move fast while handling the weighty payload. Because of the flexibilities at its joints, the residual vibration is observed after fast motion. The joint flexibility is caused by the harmonic drive which is used as a transmission device.

Suppressing the residual vibration of axis 1 is an issue, because the vibration is the most serious among all joints. In order to suppress the residual vibration the IST was applied, but the residual vibration could not be suppressed. It seems to be due to the nonlinear and time varying characteristics of the vibration.

The residual vibration has nonlinear characteristics that the frequency of the vibration decrease at the small amplitude. It seems that the characteristics is caused by the nonlinear flexibility of the harmonic drive. The vibration also has the time varying characteristics. Because all joints of this

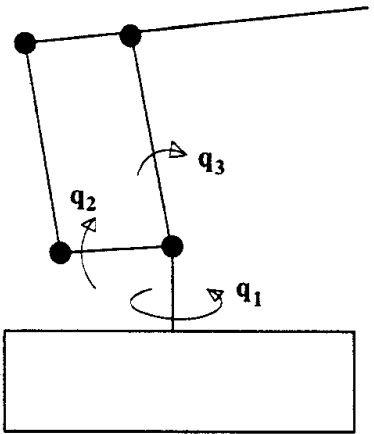

Figure 7: The schematic of the six-D.O.F robot which is used for experiment: the axes of wrist were omitted.

robot move at the same time, the configuration of the robot changes during the motion. Therefore the vibration of axis 1 depending on the configuration of the robot is considered to be time varying.

A schematic diagram of the experimental setup is shown in Fig. 8. Reference trajectories for all axes are designed to have a trapezoidal velocity profile. The position controllers are $\mathrm{P}$-controllers which have the inner PI velocity control loops. The residual vibration is measured by a accelerometer installed at the tip position. The IST is implemented in the robot controller while the parameters of the IST are imported from an outer device. The learning process is done in PC, IBM 486DX2-66.

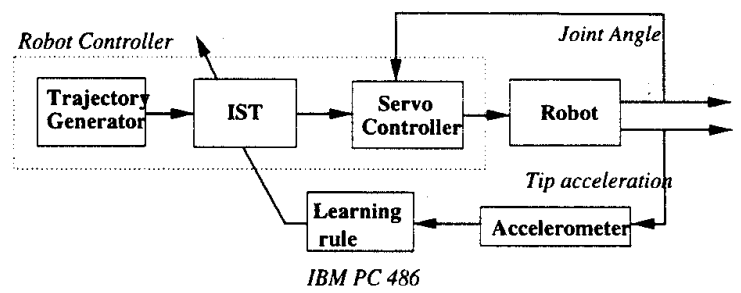

Figure 8: Experimental setups used for experiment of learning input shaping technique. The lerning process is done on the IBM-PC.

While the robot is moving, the acceleration signals measured by the accelerometer are sent to the $\mathrm{PC}$. This signals are filtered with a high pass filter in order to filter out the drift signal. Then the signals are stored in the memory of the PC. After the 1-cycle of motion, the PC calculates the $M$ and $\phi$, and then updates the IST parameters. The values of the parameters updated are sent to the robot controller, then the next cycle is started.

Experimental results are shown in Fig. 9 - Fig. 11. Fig. 9 shows the residual vibration without IST. Since the robot moved for $1.0 \mathrm{~s}$, the tip acceleration after $1.0 \mathrm{~s}$ is the residual 
vibration. Fig. 10 is the plot of the tip acceleration signals which are filtered with high a pass filter of $2 \mathrm{~Hz}$. Fig. 11 shows the variation of the measure $M$ and the parameters updated by the learning scheme. With the parameters initially guessed, the magnitude of residual vibration was very large. However the residual vibration became smaller and smaller as the number of iterations increases.

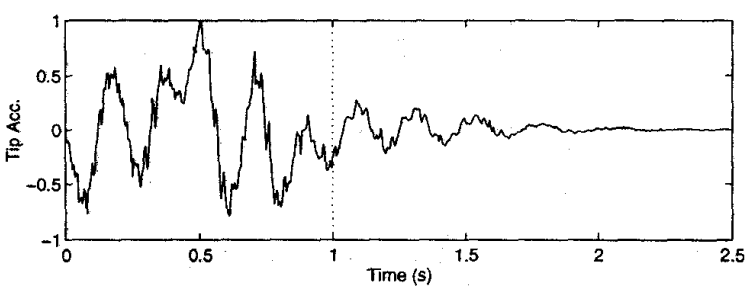

Figure 9: Experimental results: residual vibration without IST. Because the robot moved for $1.0 \mathrm{~s}$, the tip accelleration after $1.0 \mathrm{~s}$ is the residual vibration.
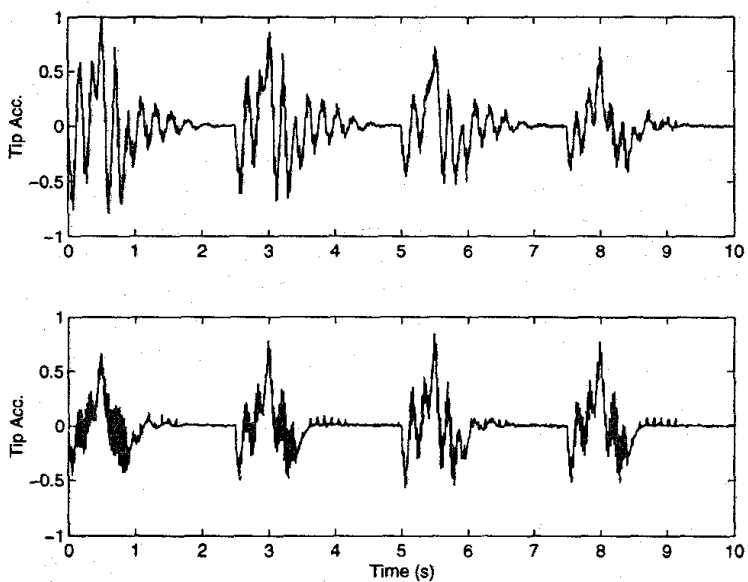

Figure 10: Experimental results: the change of residual vibrations. Residual vibrations become smaller and smaller as the number of iterations increases.

Nevertheless, the $M$ did not become 0 after sufficient numbers of iteration and it sometimes increased a little. It was caused by the high frequency noise and the characteristics of $M$ with the sharp plot near the zero in the relation of $M$ vs $T_{2}$ (or $M$ vs $A_{1}$ ).

\section{Conclusions}

The LIST is proposed to suppress the residual vibrations of nonlinear or time varying systems. In order to verify the algorithm, simulations are performed for four different cases in which the conventional IST could not suppress the residual vibration successfully. The experiments are made by using a six D.O.F industrial robot to evaluate the method.

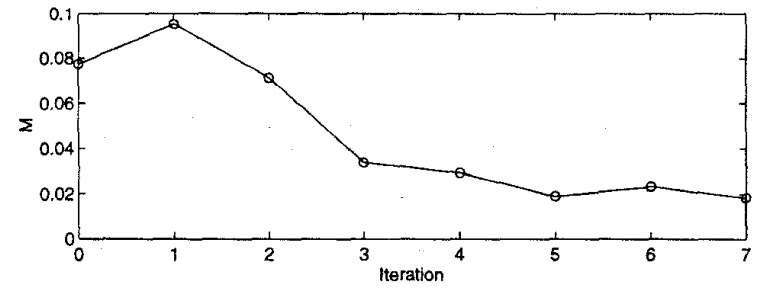

Figure 11: Experimental results: the $M$ decreses as the number of iterations increases.

The results of both the simulations and experiments show that the residual vibrations become considerably smaller as iteration goes on, thereby demonstrating the effectiveness of the LIST.

Further studies on the following areas seem to be needed to generalize the LIST scheme:

- For what categories of systems the LIST is applicable.

- Whether the relation of $M$ and $\phi$ vs parameters is acceptable in nonlinear time varying system.

\section{References}

[1] N. C. Singer and W. P. Seering, "Preshaping Command Inputs to Reduce System Vibration," ASME Journal of Dynamic System, Measurement and Control, vol. 112, pp. 76-82, 1990.

[2] N. C. Singer, "Residual Vibration Reduction in Computer Controlled Machines," Ph.D thesis, Department of Mechanical Engineering, MIT, also AI-TR-1030, The Artificial Intelligence Laboratory, Massachusetts Institute of Technology, Cambridge, Mass, 1988.

[3] A. Tzes and S. Yurkovich, "An Adaptive Input Shaping Control Scheme for Vibration Suppression in Slewing Flexible Structure," IEEE Transactions on Control Systems Technology, vol. 1, No. 2, pp. 114-121, June 1993.

[4] T. Noritsugu, "High-speed positioning of pneumatic robot using learning posicast control," Transactions of the Japan Society of Mechanical Engineers Tokyo, Vol. 59, No. 563, June, pp. 1779-1786, 1993.

[5] M. Bodson, "An adaptive algorithm for the tuning of two input shaping methods," Proceedings of the 1997 American Control Conference, 1997.

[6] B. W. Rappole, "Minimizing Residual Vibrations in Flexible Systems," Master thesis, MIT AI lab., 1992.

[7] D. P. Magee and W. J. Book, "Eliminating Multiple Modes of Vibration in a Flexible Manipulator," Proceedings of the 1993 IEEE International Conference on Robotics and Automation, Atlanta, GA, May 2-7, 1993.

[8] D. P. Magee and W. J. Book, "Implementing Modified Command Filtering to Eliminate Multiple Modes of Vibration," Proceedings of the 1993 American Control Conference, pp. 2700-2704, June 1993. 